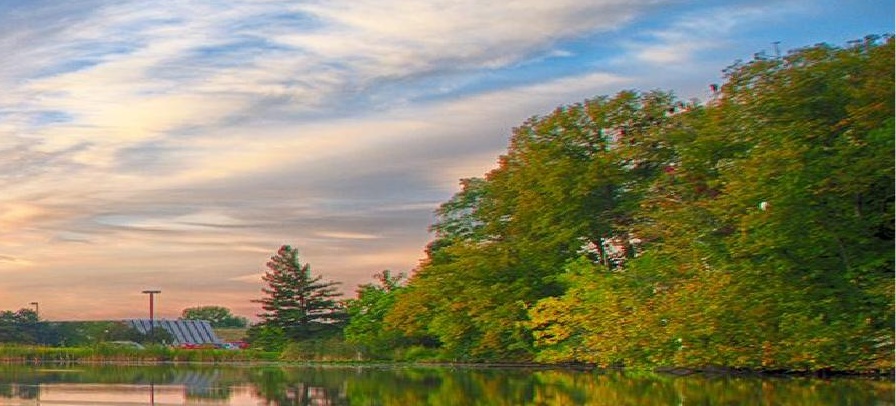

\title{
Updating Hadron Models to Better Predict Neutrino Flux for DUNE
}

Ethan Tuttle

Supervisor: Leo Aliaga

SIST Presentation

August $5^{\text {th }}, 2020$

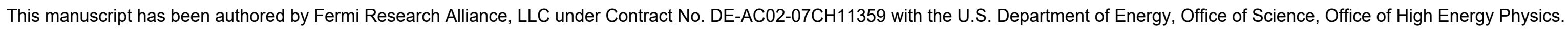




\section{A Brief Review of Neutrino Physics}

- There are 3 "flavors" of neutrinos

- Flavor is determined by associated charged lepton

- Massless in the Standard Model

- Rarely interacts

- Only interacts via the weak force and gravity

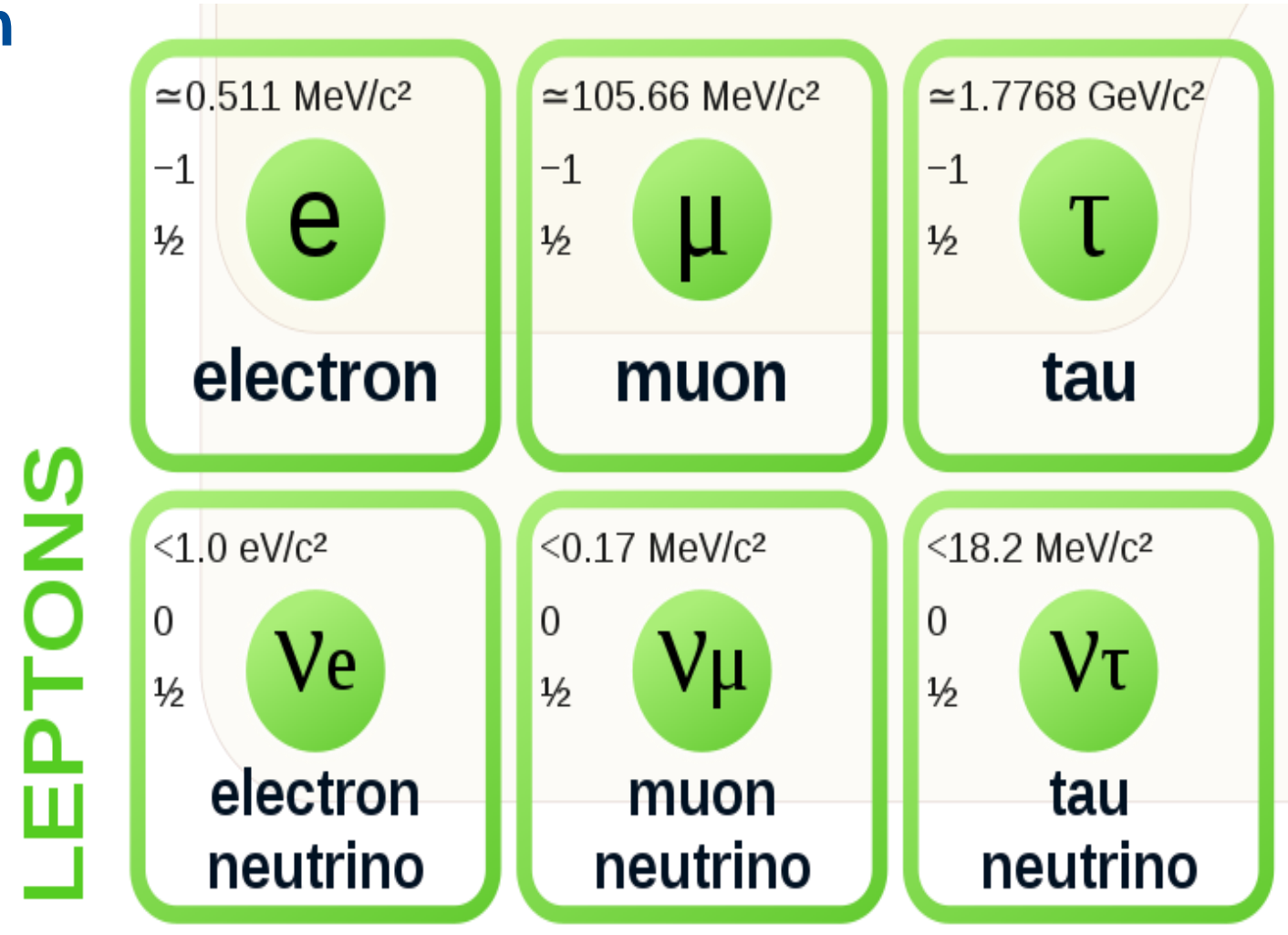




\section{Why Do We Care?}

- Why Care About Neutrinos?

- Neutrinos are everywhere

- Now believe neutrinos have mass

- Could explain why there is a matter-antimatter imbalance in the universe

- Why Care About DUNE?

- Optimized to study Charge Parity symmetry violation

- Study neutrino oscillation i.e. how neutrinos change flavor

- Look for neutrinos coming from supernovas

- Dark matter, proton decay, and more

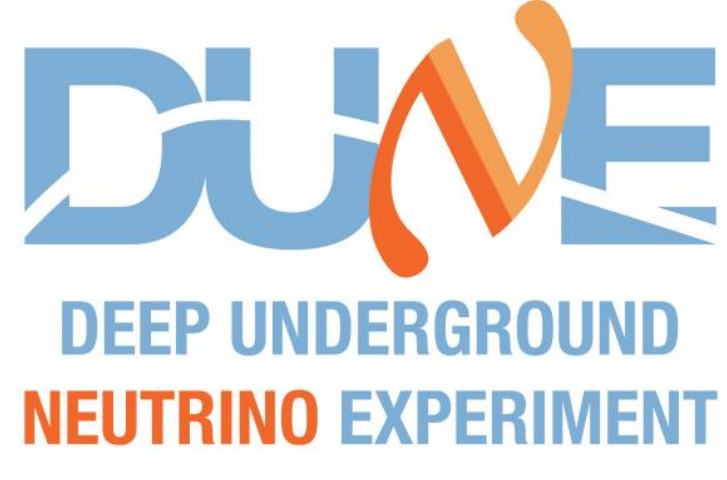




\section{LBNF (Long Baseline Neutrino Facility)}

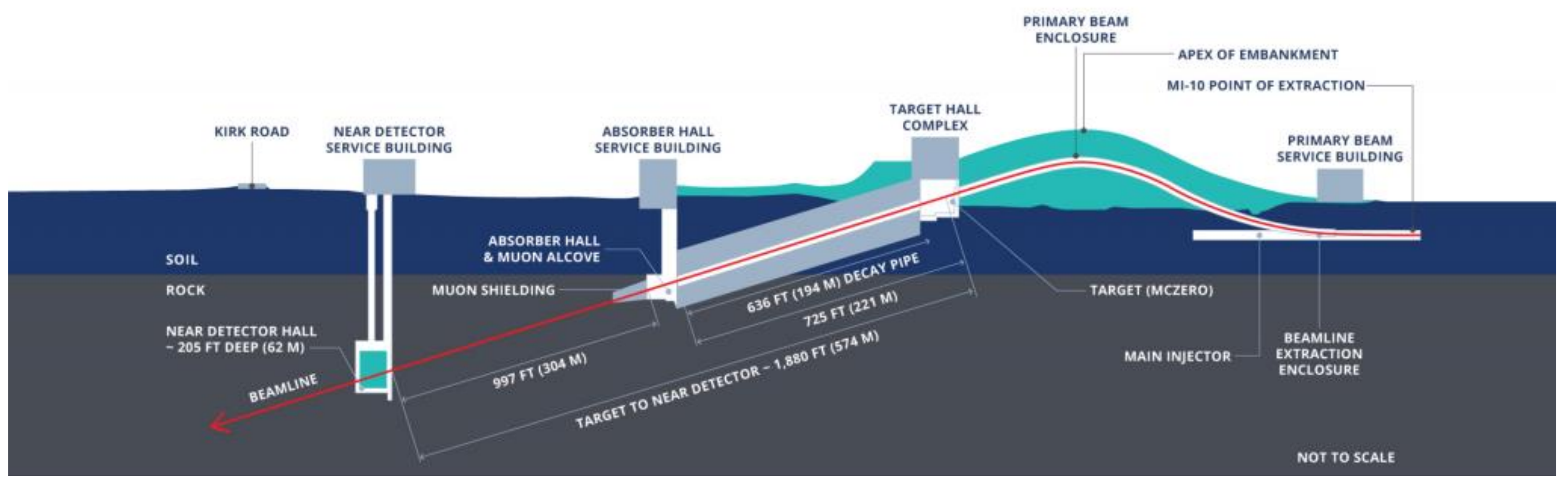

- Primary proton beam 60-120 GeV

- Beam power of 1.2 MW, upgradable to $2.4 \mathrm{MW}$

- 2 m long graphite target

- 3 magnetic horns

- Near Detector is approx. 574 meters from target, located at Fermilab

- Far Detector is approx. 1300 kilometers from target, located at Sanford Underground Research Facility 


\section{My Project}

- Characterize the hadronic model interactions and then compare with experimental data from NA49.

- Then check the effect that correcting the models with the experimental data has on the flux prediction.

- It is important for DUNE that predictions of the neutrino flux are understood and have characterized uncertainties so measurement of neutrino oscillation can be made more reliably and accurately 


\section{My Progress}

- Improved skills with Unix, $\mathrm{C}++_{+}$Root, Geant4, and learned how to use the grid

- Extracted simulated data on interactions, yields, and cross sections from Geant4

- Applied corrections to the QGSP_BERT model

- Corrections have been applied for proton on carbon creating pions and for the cumulative particles. 


\section{Muon Neutrino Flux at the Near Detector}

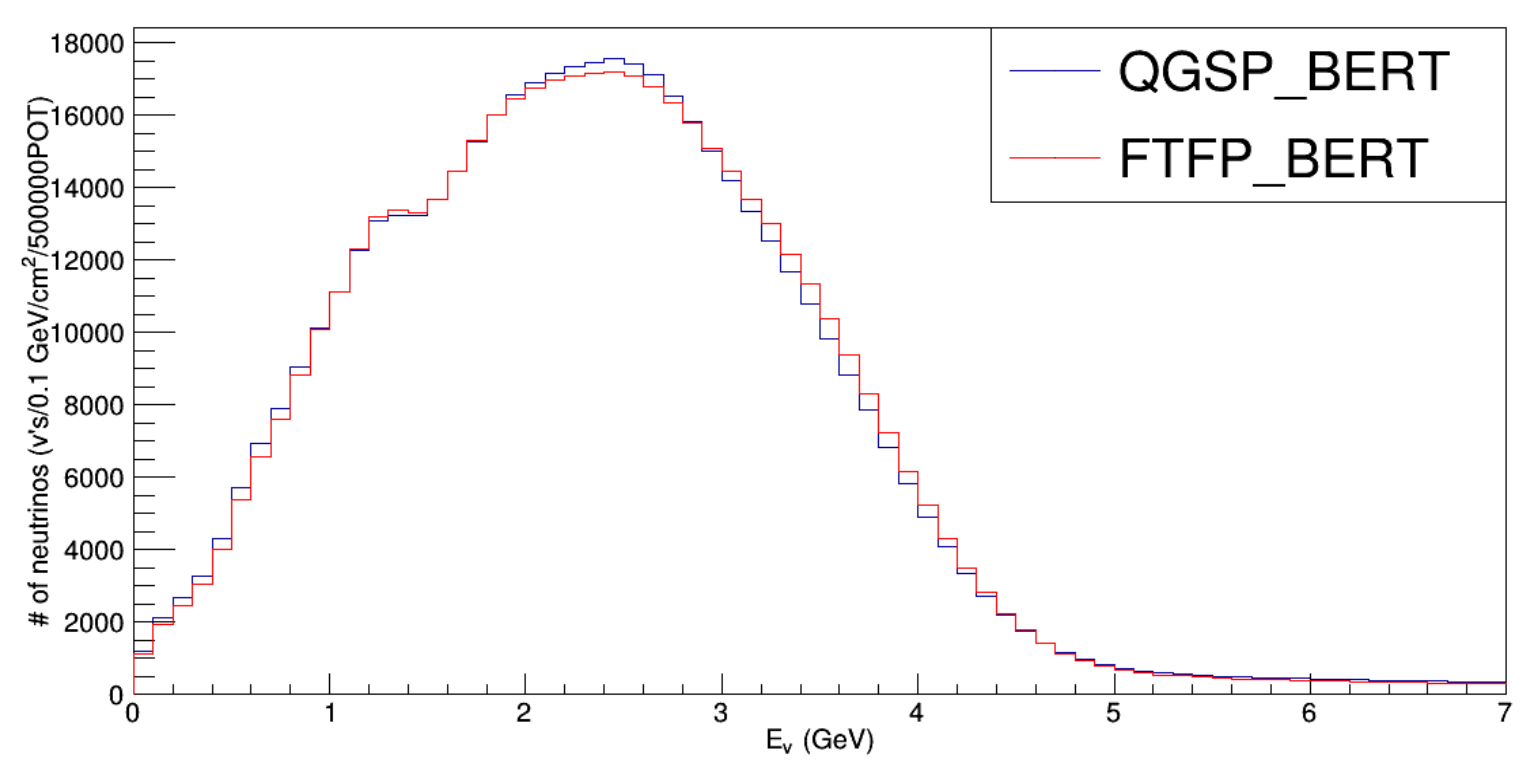

QGSP_BERT/FTFP_BERT Muon Neutrino Flux Ratio

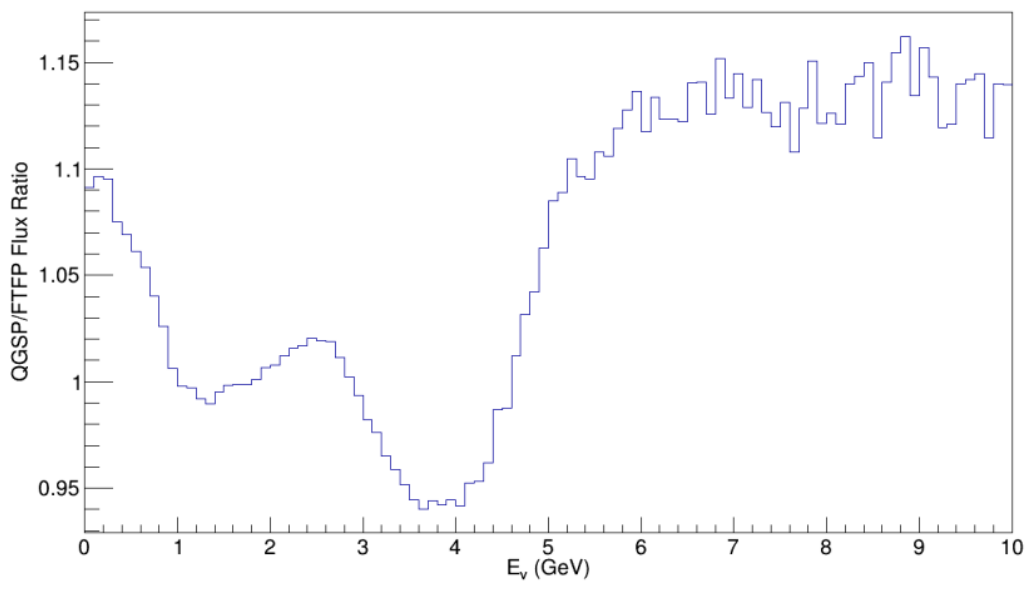




\section{Total Interactions}
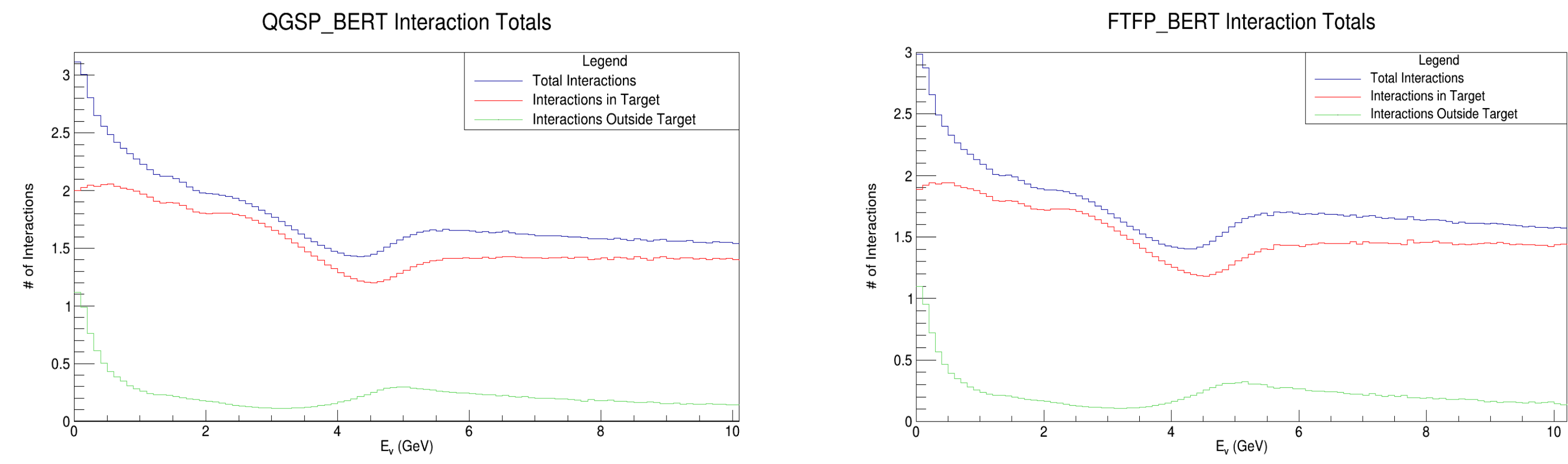


\section{Interactions in Target}
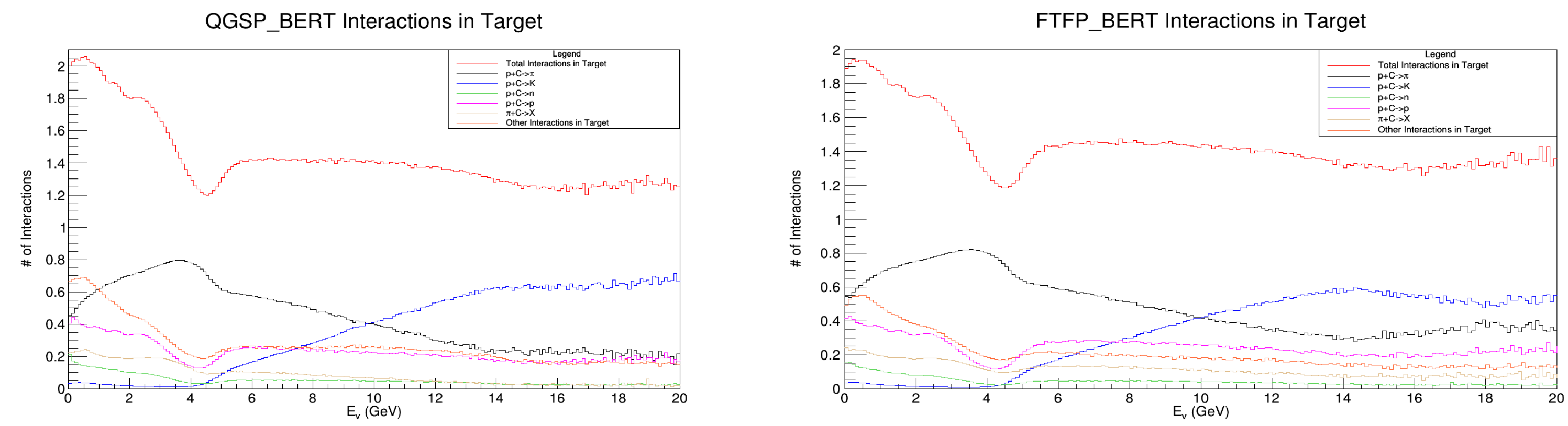


\section{Pion Kinematics}

\section{QGSP_BERT Pion Momentum}

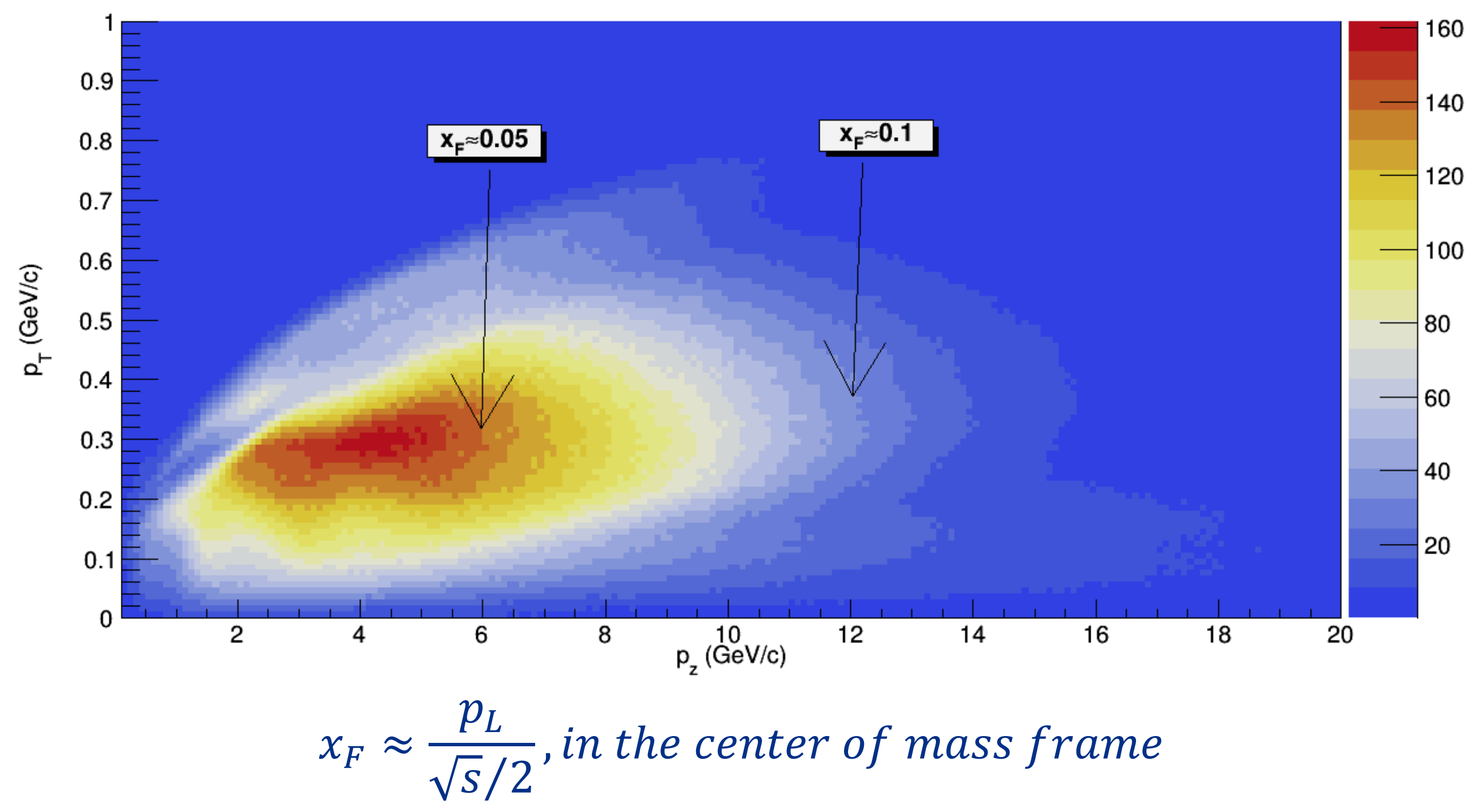




\section{QGSP/FTFP Cross-Section Ratios}

QGSP/FTFP 120GeV П+ Ratio

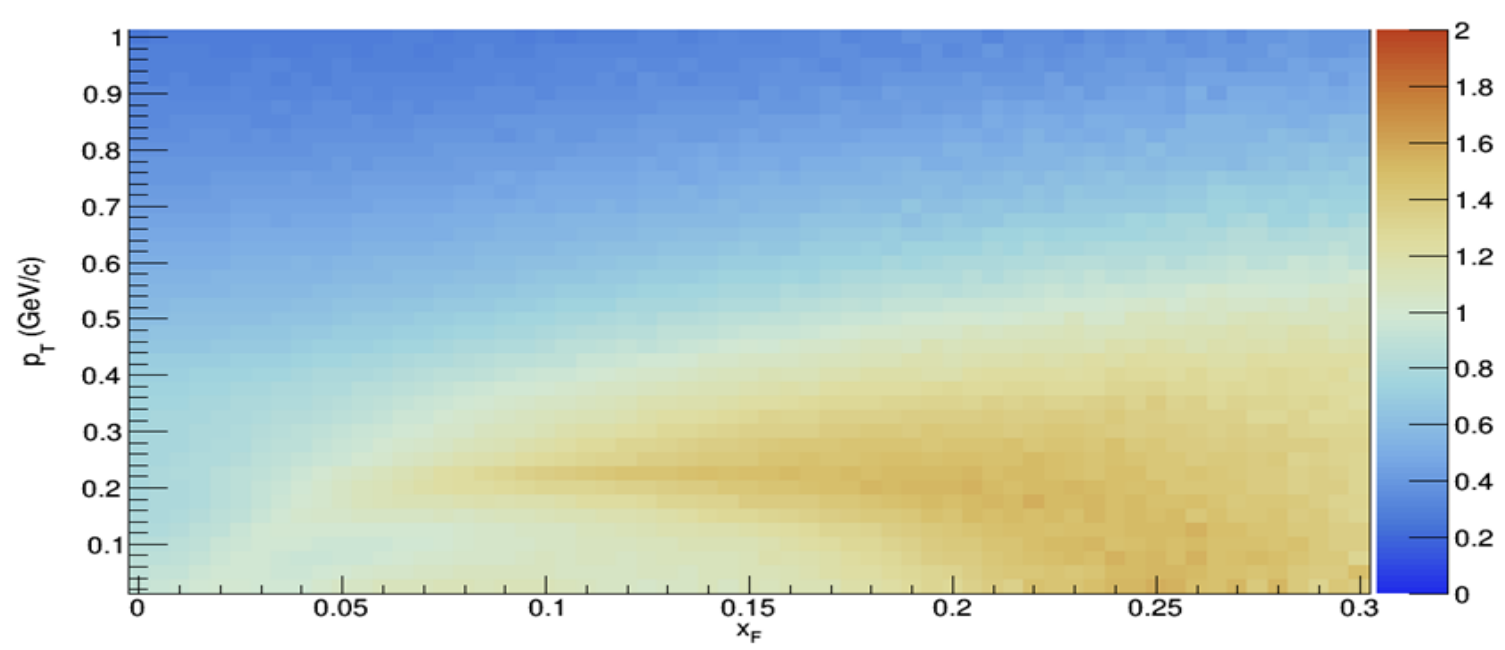

QGSP/FTFP 120GeV ח- Ratio

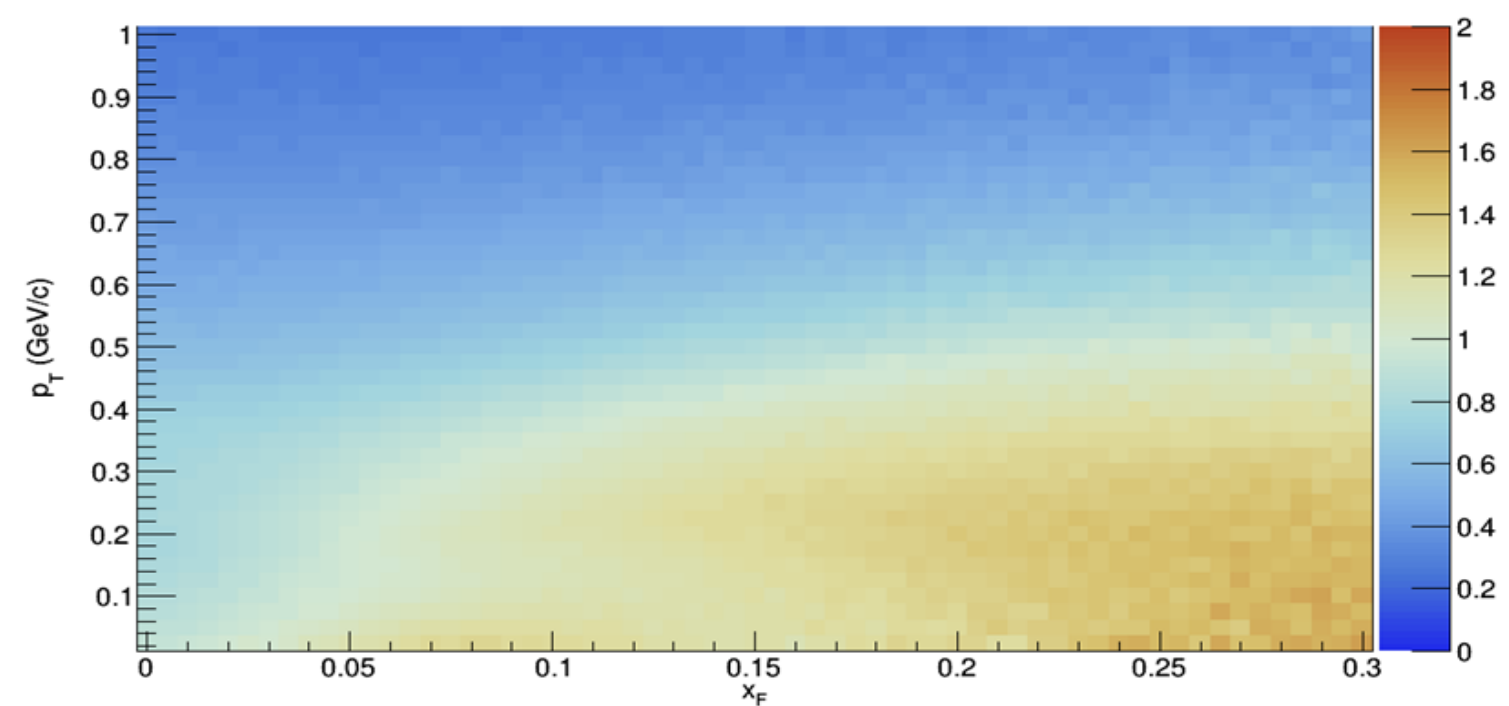

QGSP/FTFP 158GeV П+ Ratio

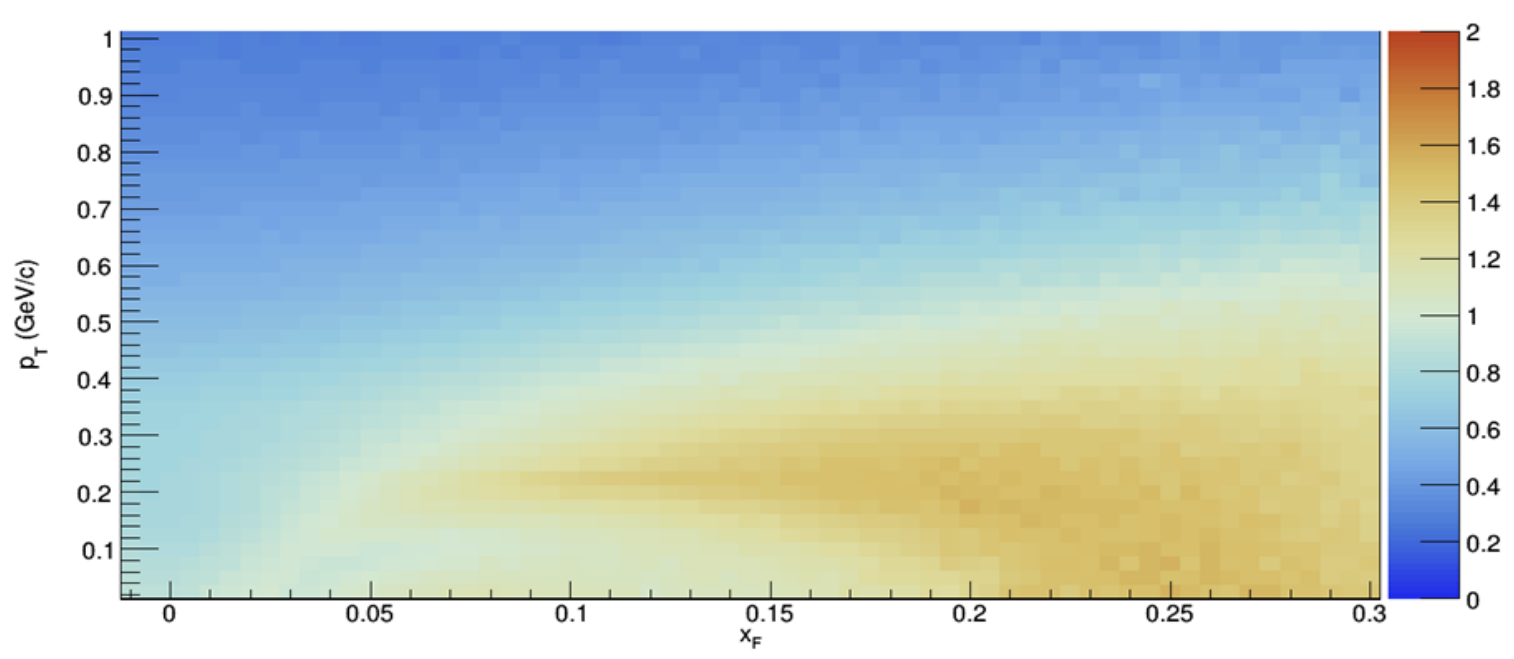

QGSP/FTFP $158 \mathrm{GeV}$ П- Ratio

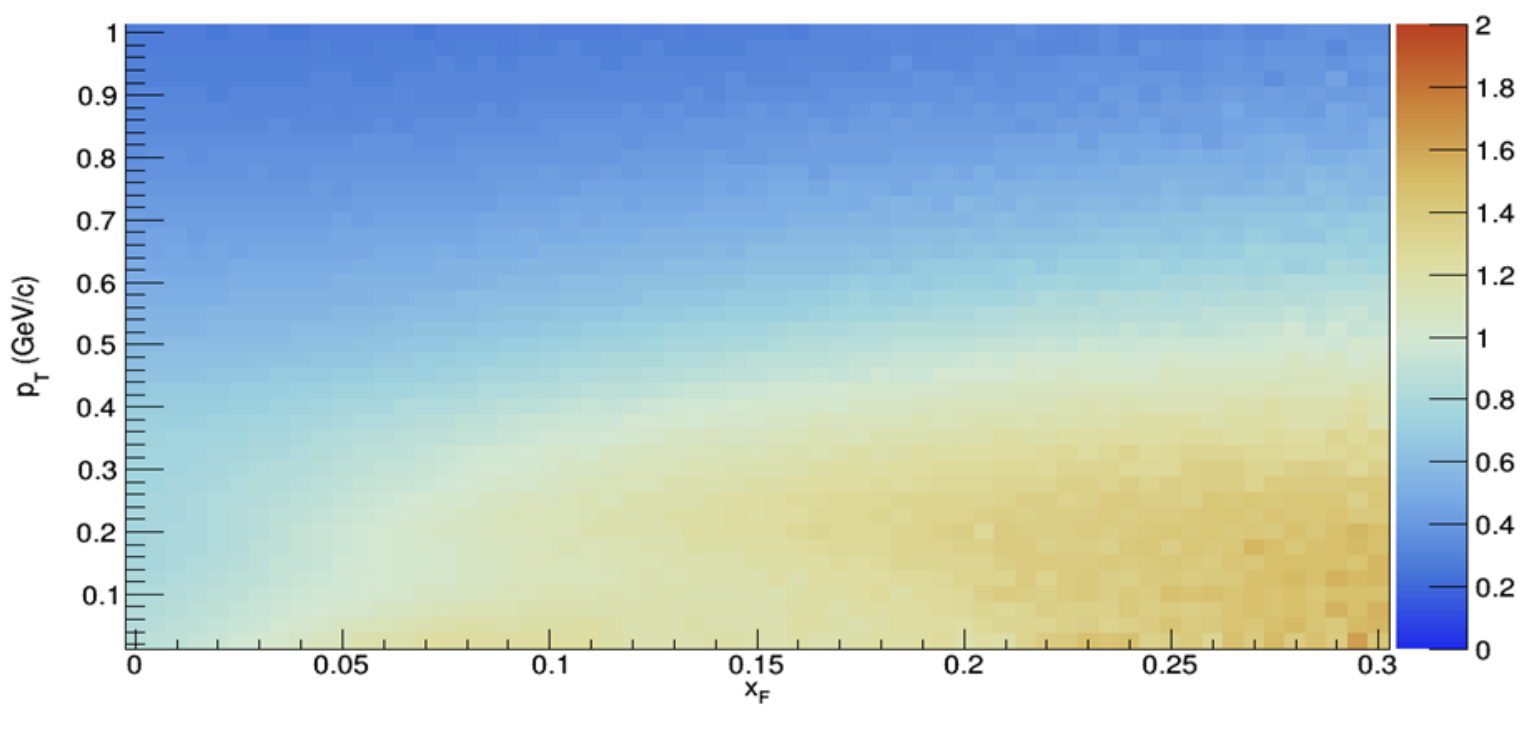




\section{NA49}

- Experiment conducted at CERN on hadron production

- Started Sept. 1991 and completed Oct. 2002

- Studied various proton interaction

- Studied proton on carbon interactions at $158 \mathrm{GeV}$

- Has Cross section data for Pions

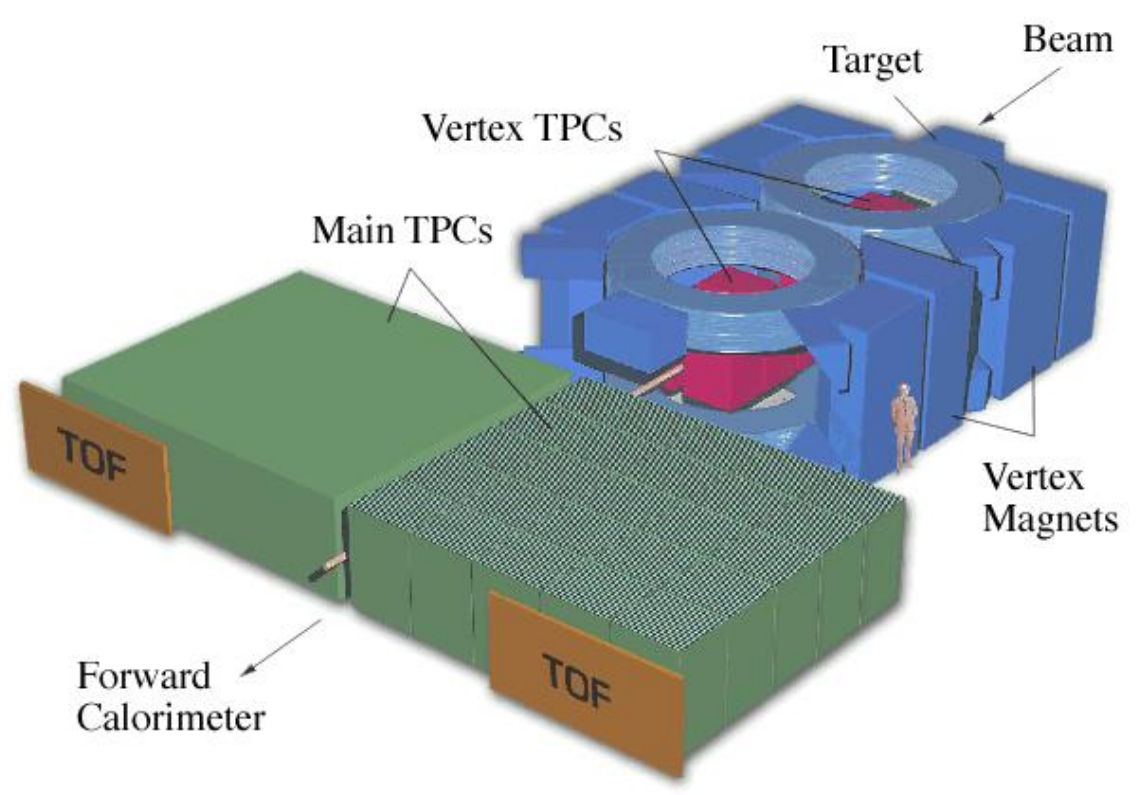




\section{Invariant Cross Section}

Cross Section at $x_{F}=0.05 \pi+158 \mathrm{GeV}$

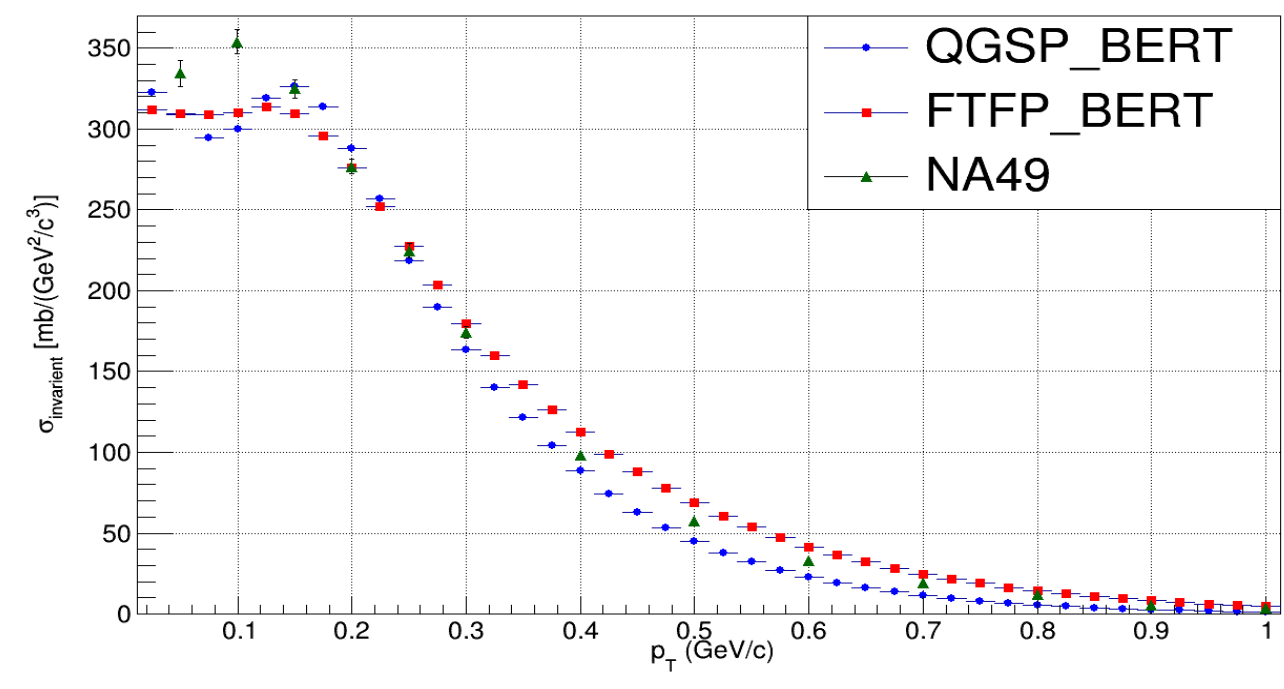

Cross Section at $x_{F}=0.1 \pi+158 \mathrm{GeV}$

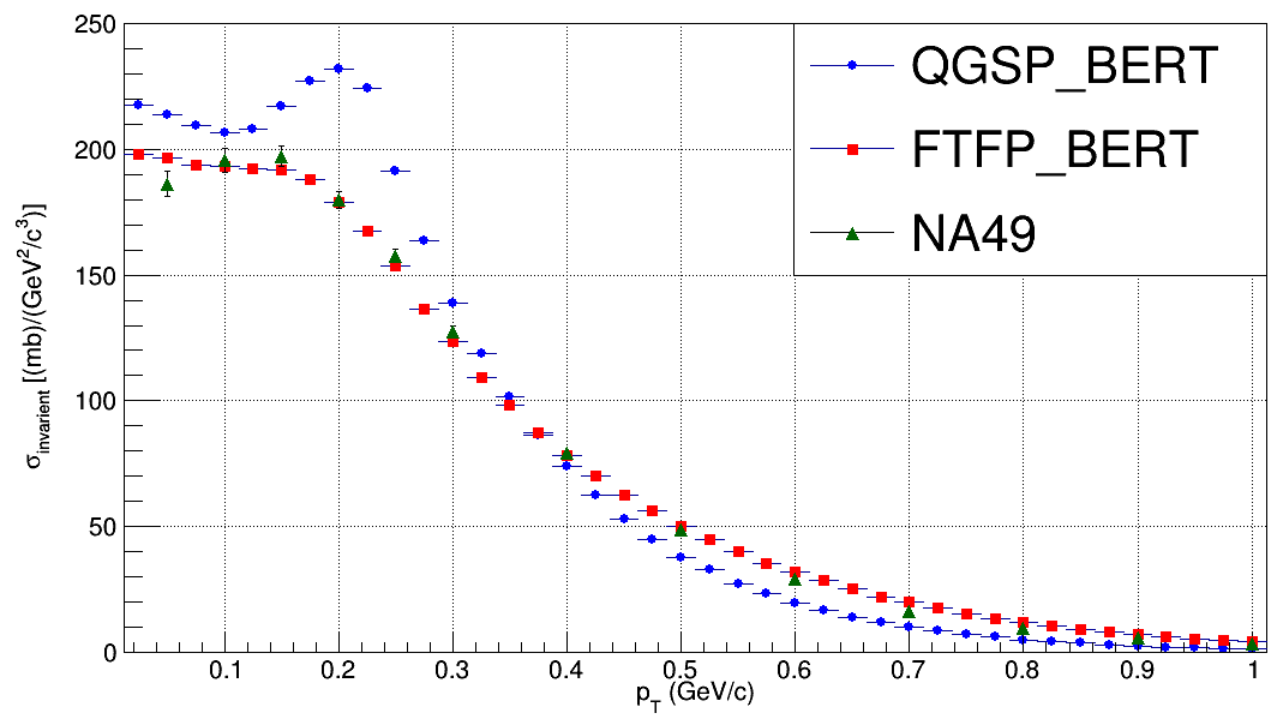

Cross Section at $x_{F}=0.05 \pi-158 \mathrm{GeV}$

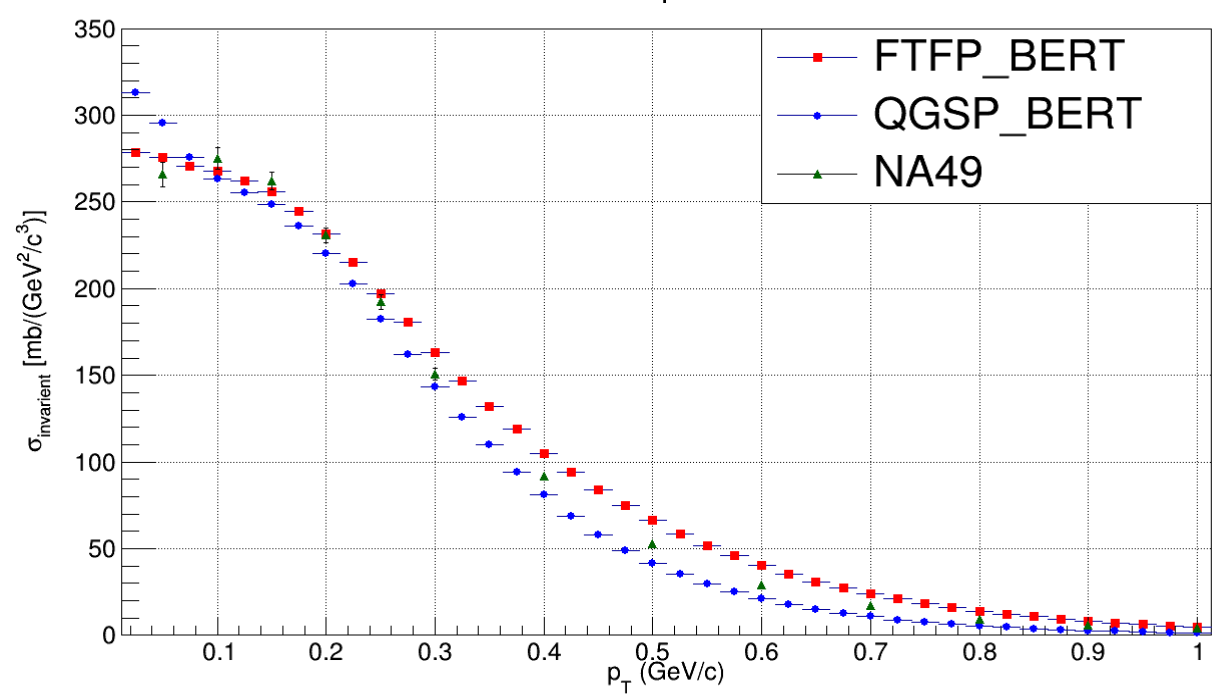

Cross Section at $x_{F}=0.1 \pi-158 \mathrm{GeV}$

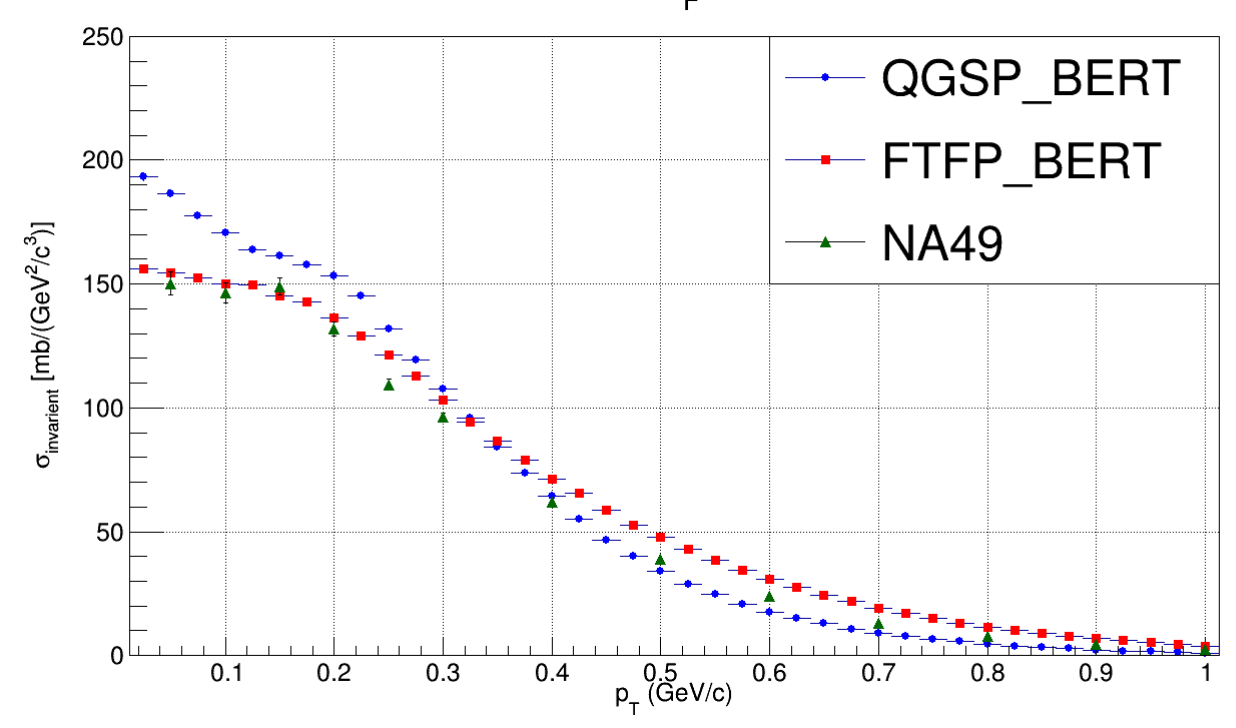




\section{Pion Production Ratio $158 \mathrm{GeV}$}

$\pi+/ \pi$ - Ratio at $\mathrm{x}_{\mathrm{F}}=0.05$

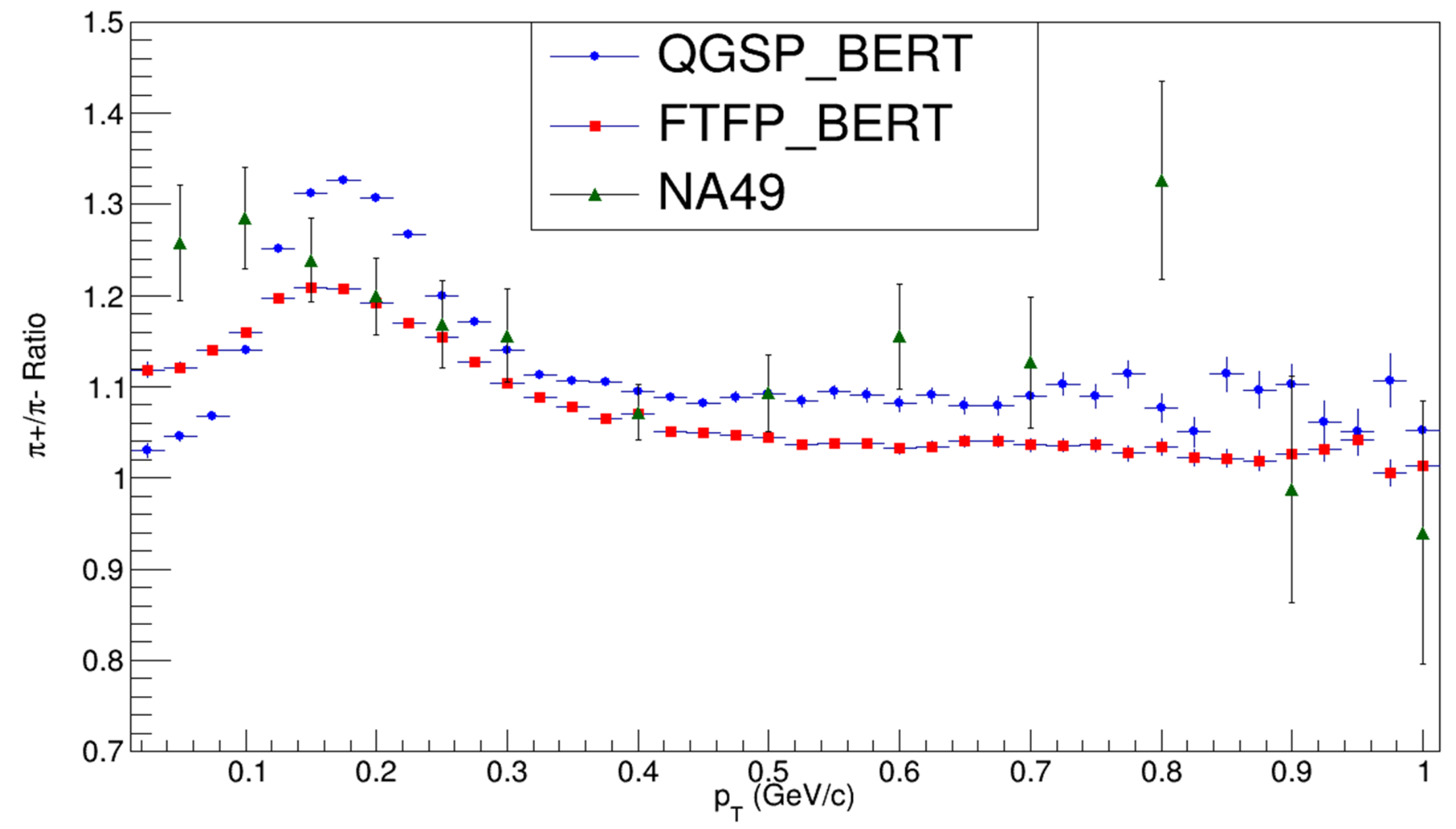




\section{Results}
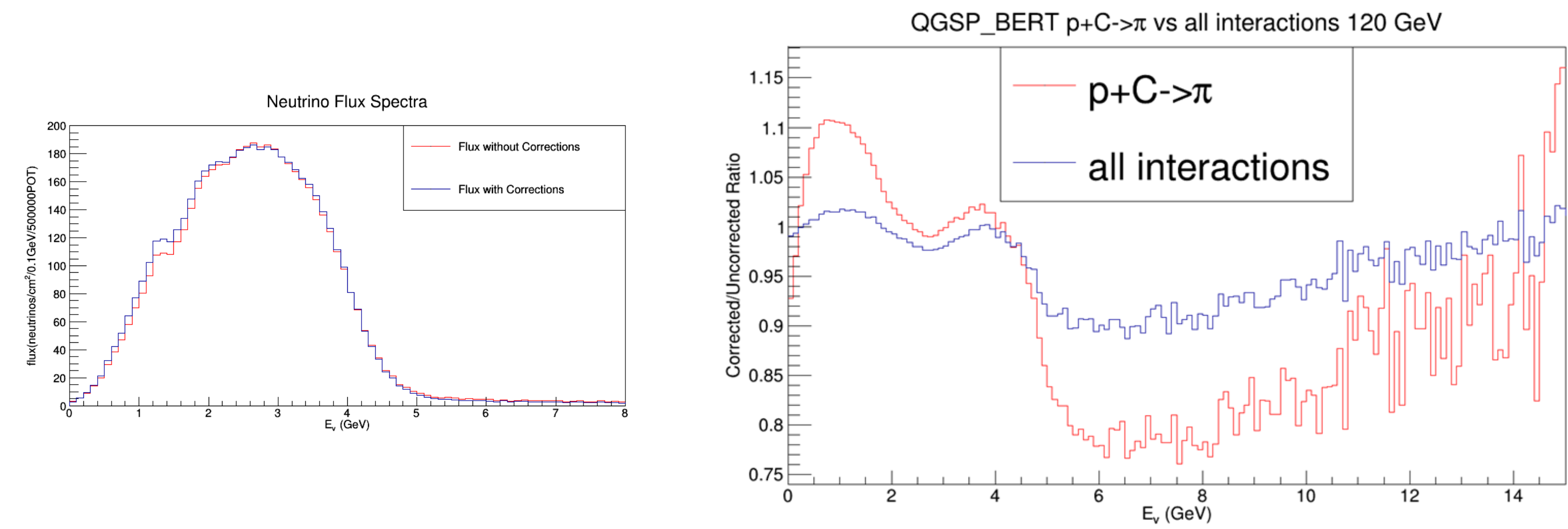


\section{Conclusions}

- When there are no corrections FTFP_BERT on average does a better job of more closely following the NA49 data than QGSP_BERT

- My project can be used as a template to correct for other particles, e.g. kaons.

- Next step should be to apply corrections beyond primary proton i.e. particles that reinteract in the target. 


\section{Acknowledgements}

- Thank you

-Supervisor: Leo Aliaga

-Mentors: Arden Warner and Charlie Orozco

-The SIST committee for transitioning this experience online 